\title{
Research on Algorithm of Civil Aircraft Network Communication Routing Across the Ocean Environment
}

\author{
Dehai Yang ${ }^{1}$, Yuqin $\mathrm{Yao}^{1, a}$ \\ ${ }^{1}$ Chengdu University of Information Technology, Chengdu, 610225, China \\ ${ }^{a}$ Email: yyq@cuit.edu.cn
}

Key words: Aeronautical ad hoc network, AODV, OLSR

\begin{abstract}
With the rapid development of modern aviation industry, the air traffic is becoming more and more advanced, modern communications technologies are required to ensure aviation safety and the efficiency of navigation. The concept of aeronautical Ad Hoc network have been proposed on these requirements, which is successfully applied to aviation aircraft .This paper based on summarization of the current situation of Ad Hoc network routing protocol, aimed at a complex environment for aviation communications, simulated and evaluated the applicability of routing protocols AODV and OLSR. Simulation results show that, AODV delay jitter is lower than OLSR, indicate AODV network stability better than OLSR. .
\end{abstract}

\section{Introduction}

Wireless ad hoc network is also known as the "Ad Hoc network", it is a multi-hop temporary autonomous system consisting of a group of mobile terminals with wireless transceivers. In ad hoc network, each user terminal can not only mobile, but also have the functions of routers and hosts ${ }^{[1]}$. On one hand, as a host, the terminal runs varieties of users' application; on the other hand, as a router, the terminal needs to run the related routing protocol, and finish the data packets and forward according to the routing policy and routing table, at the same time accomplish the routing maintenance work. Ad Hoc net is a combination of mobile communication and computer network, and is a type of mobile computer communication network. It has the following characteristics: 1.multi hop network, 2.dynamic topology, 3.distributed control, 4.equicalence, 5.temporary, 6.self-organization, 7.linited link bandwidth, 8.one-way channel, 9limited energy, 10.limited security.

Aeronautical ad hoc network is the application of mobile Ad Hoc network in aeronautical communication field, the basic idea is: a range of aircrafts can transmit information with each other, exchange their flight status, perceived information data, and connect automatic, set up a mobile Ad Hoc network. In this network, each machine is not only a transceiver, but also a router, it can use multi hop mode to make the data forward to further planes ${ }^{[2]}$. The coming of air network, not only provide security effectively, but also can make the aircraft access Internet by satellite or terrestrial base station, passengers can enjoy cyber source.

\section{Analysis of routing protocols in ad hoc network Airlines}

As the aeronautical ad hoc network (Aeronautical Ad Hoc networks, AANET) has the characteristics of large range, dynamic changes fast, temporary, no central node, the time-varying channel and node density is small, so compared with the traditional MANET on the ground, it's much more complex ${ }^{[3]}$. With the developing of aeronautical communication, there still exist many problems which haven't been solved. Among these problems, the routing algorithm is one of the most important research topics in the field, according to the different scenarios, the existing MANET routing protocols are more or less exist defects, especially in the complicated air environment, so we need to make a summary of the existing air routing protocol in ad hoc network, in order find the direction of our research. 


\subsection{AODV routing protocol}

AODV is essentially a DSR (as required) and DSDV (active) integrated, wherein, destination sequenced distance vector routing (DSDV, Destination Sequenced Distance Vector) is an improvement on the basis of DVA routing protocol ${ }^{[4]}$. It is believed to be the earliest of the routing protocol in ad hoc network. Each node in the protocol has a relatively stable new routing table, just do table look-up when communication, without the need for the route discovery. Main characteristics are use the sequence number mechanism to distinguish the old and new router table, and prevent routing loops. At the same time keep the simplicity of DVA. The disadvantage is the ad hoc network does not adapt to quick changing network, and do not support unidirectional channel. DSR (Dynamic Source Routing) is one of the first uses of on-demand routing ideological agreement. DSR features a routing mechanism uses source routing, which carries the whole routing information in each data packet header, the router forward packets according to the routing records ${ }^{[5]}$. This mechanism was first used in a token ring network bridge interconnect routing by IEEE802.5. DSR protocol uses source router and store router information with Cache, it don't have to store forward routing information required, with less network overhead, but exists old route.

The AODV algorithm also has some defects:

1 The Agreement applies only to the two-way channel;

2 In the AODV protocol, the routing table maintains only one route to the specified destination nodes, and in the DSR protocol, the source node can maintain multiple routes to the destination node;

3 Because the AODV protocol uses a timeout deleted routing mechanism, so even if the routing is failure, it will be deleted within overtime; requires a longer route establishment delay.

\subsection{OLSR routing protocol}

The OLSR protocol has two kinds of control message: Hello message and TC (Topology Control) message. The Hello message is used to find a node's neighbors. Each node broadcast Hello messages periodically, including the link state information between one hop neighbor nodes and the node information, the wireless link state information includes: symmetric link, asymmetric link and linked MPR. At the same time, the Hello message will calculate the node of MPR. Hello message only broadcast in one hop range will not be forwarded by neighbor nodes. The TC message is used for network topology formation. TC messages broadcast and forwarding in the whole network, including the node selected as MPR's neighbor information, through the forwarding of TC messages we can calculate the topology of the network. Not all the nodes in the network will forward or broadcast TC messages, it's only used in the node which is chose as MPR. Through forwarding HELLO messages and TC messages between nodes, the route table of the whole network is formed. OLSR protocol uses the MPR flooding technology. In the MPR flooding technology, only the node which is chose as MPR will broadcast and forward control messages, control message contains only the link state information between itself and the MPR node. In this way, the size of the message and forwarding scale are reduced, the control overhead is also reduced. This protocol with low delay between nodes is especially suit for real-time information transmission fields. But when the network size increases, the node density increases, MPR node gradually increased, TC messages will spend a lot of cyber source as forwarding in the network, in addition, the TC message will increase as the number of nodes increases, comes with the conflict and collision problems between messages, the ratio of the successful news decreases, and the network does not have the extendibility.

The core of OLSR protocol is the multipoint relay (Multipoint Relay) technology. The idea of multi point relay is reduce frequency of the same control packet in the same area to reduce the number of broadcast packets. Every node in the network chooses one subset of the neighbor node to transmit control packets. This chosen node is called MPR. And the node itself is called relay node (MPR Selector). The MPR of node $\mathrm{N}$ is chose from binary link neighbor, thus, through multi router, avoid transmit data packets in a single link automatic. The decrease of link state information and multi relay station can cut down the overhead of the router. 


\section{Performance of AODV and OLSR} jitter.

In our study, we mainly analyze the performance of two routing protocols through the delay

The state of the network is changing at any time, traffic of network is unstable, when the flow is large, and many packets wait in the queue delay nodes, so the delay in each packet in the transmission process is not consistent. The delay jitter of $\mathrm{J}$ (Jitter) is used to describe the changing of delay; the network is less stable if the delay jitter is large. In some network applications, such as the need for real-time information communication occasions, more attention is paid than transmission delay, although the average delay time may be very small, if the delay jitter is large, it will have a direct impact on both sides of communication. For applications like network television which uses multicast or broadcast, as long as the general average broadband transmission is greater than the video playback rate, it can be achieve real time; if the delay jitter is large, you will need to do a large buffer before play to absorb the jitter caused by transmission, otherwise it will cause the picture discontinuous and have impact on the quality of the video.

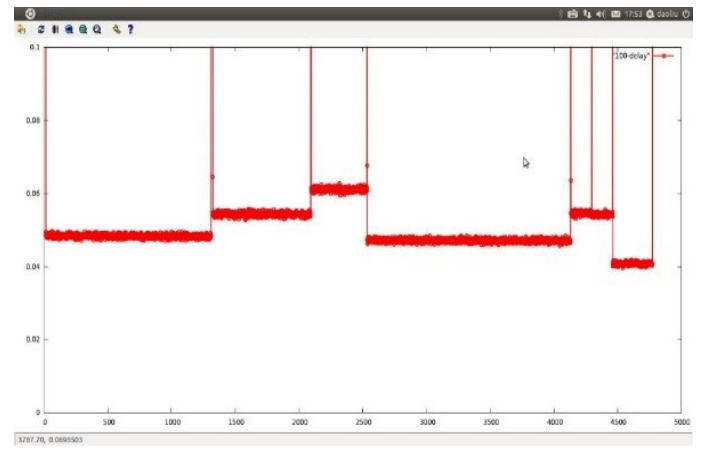

Figure 3.1 delay of AODV

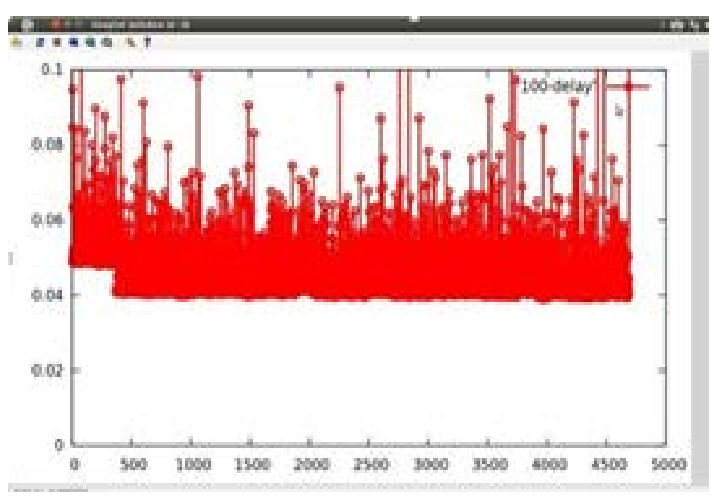

Figure 3.2 delay of OLSR

In our research, through analyise of trace file, we define delay jitter as the difference of the two groups before and after, it's computational method is:

$$
J(i)=(D(i)-D(i-1))
$$

After write down the gawk program, the simulation is as follows:

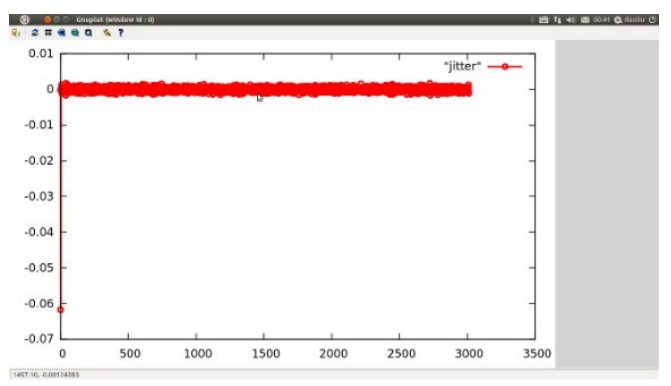

Figure 3.3 delay jitter of AODV

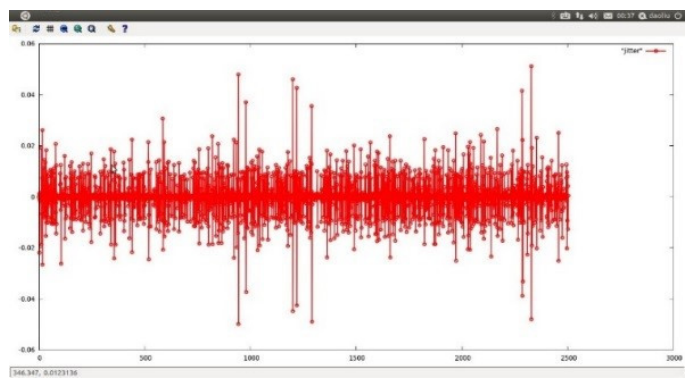

Figure 3.4 delay jitter of OLSR

In the two figures, the abscissa represents the simulation time (unit is ms), the ordinate represents the delay jitter (unit ms). The node speed in the two figures is all $100 \mathrm{~m} / \mathrm{s}$. Through comparison, we know the jitter range of AODV is in $(-0.01,0.01)$, but the jitter of OLSR is in range of $(-0.06,0.06)$. It's obvious; in this experiment the stability of AODV is better than OLSR. Through other simulation, we know that the stability of AODV is also better than OLSR with other speed. 


\section{Conclusion}

In this paper, based on the previous research of aircraft routing protocol, after continuous learning, we compared and classified the aircraft protocol. We mainly analyzed and compared the delay jitter of AODV and OLSR protocol. In the aviation environment, the stability of AODV is better than OLSR, the delay of AODV is longer than OLSR.

In this research, there are some shortcomings, first the scene is very simple, only one UDP link and CBR data stream. Second is the data of simulation is not enough, for example, we didn't set routing hops to change the simulation, no simulation of the network throughput, these lead to the route algorithm is not thorough in aviation environment. For the particularity of the aviation environment, in order to facilitate the simulation, we need to choose a reasonable setting, and after the scene and parameters is set, the performance of the network will be best, and then find out the suitable routing algorithm of civil aviation.

\section{References}

[1] Nash JF.Equilibrium points in N-person games. Proceedings of the National Academy of Sciences of the United States of America . 1950

[2] D.K.Gode,S.Sunder."Double Auction Dynamics:Structural Effects of Non-Binding Price Controls,". Journal of Economic Dy-namics and Control . 2004

[3] Sakhaee E,Jamalipour A.The global in-flight internet. IEEEJournal on Selected Areas in Communications . 2006

[4] Niyato D,Hossain E.Competitive pricing for spectrum sharing incognitive radio networks:Dynamic game,inefficiency of Nashequilibrium and collision. IEEE Journal on Selected Areas inCommunications . 2008

[5] Jitendra Padhye,Victor Firoiu,Don Towsley,Jim Kurose. Modeling TCP throughput[J]. ACM SIGCOMM Computer Communication Review . 1998 (4) 